\title{
TRANSVERSE RESISTIVE WALL INSTABILITY OF AN OFF-AXIS RIBBON BEAM IN A CIRCULAR CHAMBER
}

\author{
E.D. Courant and M. Month
}

June 1978

BROOKHAVEN NATIONAL LABORATORY

ASSOCIATED UNIVERSITIES, INC.

UNDER CONTRACT NO. ER-76-C-02-0016 WITH THE

UNITED STATES DEPARTMENT OF ENERGY 
BHL 50875

UC-28

(Particle Accelerators and High-

Voltage Machines - TID-4500)

\title{
TRANSVERSE RESISTIVE WALL INSTABILITY OF AN OFF-AXIS RIBBON BEAM IN A CIRCULAR CHAMBER*
}

\author{
E.D. Courant and M. Month
}

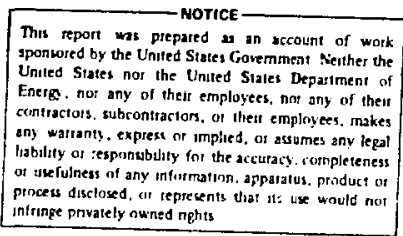

June 1978

*Lecture Series on the ISABELLE Project given by M. Month af Brookhaven Mational Laborafory, June 13, 14, and 15, 1978. 


\section{ABSTRACT}

HAgh energy proton storage rings are designed to make maximal use of the available vacuum chamber aperture. This is dictated primarily by economic considerations. The accumulation of current in a typical high energy ring creates a rather unusual beam configuration : in particular, a ribbon beam in a circular chamber set well off the central axis. The result is an enhanced resistive wall instability. This could be anticipated from the strong dependence of the current threshold on the chamber radius for a centered beam. For an offcentered beam, one might expect that the relevant replacement for the radius would be the "distance of closest approach". The case of a wide ribbon-type beam is treated. The current threshold is obtained as a solution to a "coupled oscillator eigenvalue problem". When the beam is "smal1" as occurs near the beginning of current stack formation, the problem is reduced to a dispersion relation. Results are expressed in terms of ISABELLE parameters. Some conclusions relevant to the ISABELLE design are presented. 
ISA $78-12$

\section{INTRODUCTION}

High energy proton storage rings are deslgned to make maximal use of the avallable vacuum chamber aperture. This is dictated primarily by economic considerations. The accumulation of current in a typical high energy ring creates a rather unusual beam configuration. In particular, we can have a ribbon beam in a circular chamber set off the central axis toward one side of the chamber in the median plane. It might be anticipated that such a condition could produce an enhanced resistive wall instability. Since the threshold is a strong function of the chamber radius, we could even guess, a priori, that for an offcentered beam, the threshold would significantly decrease and be roughly related to an "effective radius" which is simply the distance of closest approach to the chamber.

To use the chamber aperture optimally for high current accumulation, we would like to be able to position the beam as close as possible to the chamber on one side and continue to complete the ribbon with continued addition of injected pulses. The standard approach to the resistive wali instability is not adequate here since it essentially assumes a point beam situated at the chamber center. To provide a better description of the off-centered wide beam, we could simply average over the beam distribution to determine a "mean" position in the vacuum chamber. However, since the induced fields are sensitive to the distance of the beam, even just a part of the entire beam, to the chamber wall and since we are concerned with a wide beam where part is bound to be near the wall, we have opted not to average. Rather, we keep the full Green's function, thus allowing one part of the beam to couple to another part through the wall image fields. The major implication is that we are confronted with an integral equation for the oscillation amplitude, with the coherent oscillation frequency being determined in the process of solving a "coupled oscillator eigenvalue problem". The reduction to a simple dispersion relation for the coherent frequency does not occur when beam coupling is taken into account.

In Section 2, we review the resistive wall instability theory. We derive the Green's function, which describes the effect of one part of the beam on another via the induced image fields. We give the integral equation, showing the reduction to a dispersion relation for the coherent frequency in the case of a point beam within the chamber aperture. We then describe a method of solving the integral equation by function interpolation; and in particular, we express the solution in terms of an eigenvalue problem for the threshold current.

In Section 3, we show some results. We find, as expected, that the instability threshold is extremely sensitive to the mean beam position from the wall and is equally sensitive to the distance between the wall and the nearest beam edge (i.e., to the mean beam center and the beam vidth as well). We also find an asymetry in that a beam position on one side of the chamber is more harmful than on the other side. This is the result of the unstable coherent 
frequency, the so-called "slow wave", occurring at one edge of the beam, say the low frequency edge of the frequency distribution. Since frequency and beam position are correlated, we can hold down the impact of the instability by keeping the low frequency edge of the distribution close to the chamber center.

In the case of positive chromaticity (meaning that increasing betatron frequency implies increasing momentum, i.e. movement from inside to outside of the vacuum chamber) the high frequency stack edge is located at the high momentum edge or on the side of the beam close to the outside of the ring. The implications for stacking are thus clear: "Keep the low frequency edge of the stack away from the wall". In other words, "inject on the chamber inside and accelerate the injected beam towards the stack situated on the chamber outside".

In presenting results, we use ISABELLE parameters. Some conclusions regarding the resistive wall instability for off-centered beams and, in particular, for the ISABELLE design are given in Section 4.

\section{THEORY OF THE RESISTIVE WALL INSTABILITY}

\section{A. Electromagnetic Fields}

We treat the case of vertical coherent oscillations for a thin ribbon beam which can be displaced horizontally in the vacuum chamber. We use the Green's function method. So, consider a source as depicted in Figure 1: an infinitesimally thin (horizontally) charge at position

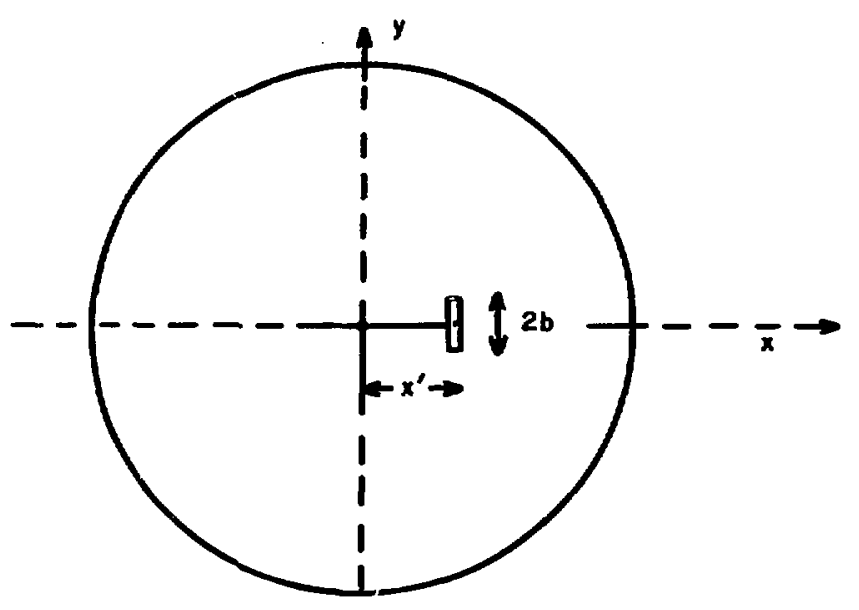

Figure 1. Geometry and source of fields. 
$x^{\prime}(y=0)$ and height 2h. Let $s$ be the coordinate perpendicular to the $(x, y)$ plane. The fields we are interested in are those resulting from a coherent vertical oscillation of the beam in the form of a wave traveling in the $s$ direction. In other words, we have at time $t$ and fixed position s, a vertical displacement $\Delta y$, given by:

$$
\Delta y=\xi\left(x^{\prime}\right) e^{i(k s-\omega t)},
$$

with $\omega$ the coherent frequency, $k$ the wave number and $\xi$ the oscillation amplitude which is a function of position in the chamber, the source point $x^{\prime}$.

The normalized unperturbed charge distribution is:

$$
\rho=\frac{I}{B c(2 h)} \delta\left(x-x^{\prime}\right)[\theta(y+h)-\theta(y-h)],
$$

where $I$ is the beam current, $P$ is the particle velocity in units of $c$, and $\delta$ and $\theta$ are delta and step functions respectively. The current density is simply

$$
j_{s}=\beta_{c} \rho
$$

If we take the total charge distribution, including the perturbation (1), then subtract the unperturbed charge, we obtain, to first order in $\xi$, the perturbed charge density

$$
\bar{\rho}=\frac{I}{B c(2 h)} \xi\left(x^{\prime}\right) e^{i(k s-\omega t)} \varepsilon\left(x-x^{\prime}\right)[\delta(y-h)-\delta(y+h)]
$$

The perturbed current density is:

$$
\overline{\mathbf{j}}_{\mathrm{s}}=\beta \mathrm{c} \overline{\mathrm{p}} \text {. }
$$

Thus, our source model is a simple plane wave traveling along the top and bottom of the ribbon beam.

We can simplify the problem of finding the fields by recognizing that the frequency $\omega$ will be low for transverse beam oscillations. Thus, in first approximation, we can neglect displacement currents. of course, when wall resistivity is included, this is not a valid approach. Thus, we take the following course: neglect displacement currents to solve the case of a perfectly conducting boundary; then reintroduce the time derivatives to include the effect of finite wall resistivity.

Perfectly Conducting Walls. For the case of perfectly conducting wal1s, we have a static problem. Maxwe11's equations are

$$
\begin{array}{ll}
\nabla \times E=0, & \nabla \times B=\mu \bar{j}_{s} \hat{t}_{s}, \\
\nabla \cdot E=\bar{p} / \epsilon, & \nabla \cdot B=0 .
\end{array}
$$

with $\hat{t}_{s}$ a unit vector alng the beam axis. 
The problem separates into independent electrostatic and magnetostatic ones. We introduce the scalar and vector potentials:

$$
\begin{aligned}
E=-\nabla V, & B=\nabla \times A, \\
\nabla & A=0 .
\end{aligned}
$$

We, therefore, obtain the equations,

$$
\begin{aligned}
& \nabla^{2} \mathrm{~V}=-\bar{\rho} / \epsilon \\
& \nabla^{2} A_{s}=-\mu \overline{j_{s}}=-(\beta / c) \frac{\bar{e}}{\epsilon} .
\end{aligned}
$$

For a perfectly conducting surface, we have the boundary conditions that the tarigential component of $E$ and the normal component of $B$ are zero: that is,

$$
\begin{aligned}
& E_{\theta} \leadsto \frac{\partial V}{\partial \theta}=0, \\
& B_{r} \sim \frac{\partial A}{\partial A}=0 \text { on the boundary. }
\end{aligned}
$$

The electric and magnetic problems are identical, with the s component of the vector potential related to the scalar potential by

$$
A_{S}=(B / c) V \text {. }
$$

Recall that the sources for the flelds are simply two points. Therefore, we can use the method of inages. Consider a complex plane

$$
z=x+i y \text {. }
$$

For a source at $z=z_{1}$, the image is located at

$$
z \text { (image) }=\frac{b^{2}}{\left|z_{1}\right|^{2}} z_{1} \text {, }
$$

as indicated in Figure 2.

If we let,

$$
z_{1}=x^{\prime}+i h,
$$

then the potential is the difference of two terms, a source at $z_{1}$ and one at $z_{1}^{*}$ :

$$
V=-\frac{I z_{0} \xi e^{i(k s-\omega t)}}{4 \pi \beta h}-\left[\ln \left|\frac{z-z_{1}}{z-b^{2} / z_{1}^{*}}\right|-\ln \left|\frac{z-z_{1}^{*}}{z-b^{2} / z_{1}}\right| j,\right.
$$

which satisfies the boundary condition, $\partial v / \partial \theta=0$ at $|z|=b$. The vector poten iial $A_{S}$ is given by (8). From $V$ and $A_{s}$, the electric and magnetic fie!ds $E$ and $B$ is be found from (5). $z_{0}$ is tha impedance of free space. 


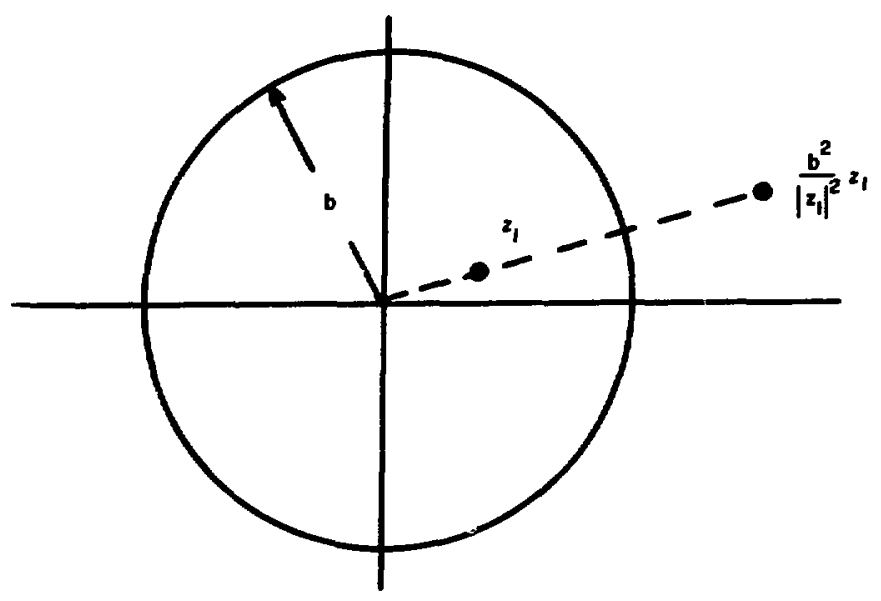

Figure 2. z-Plane for solution by images.

\section{B. The Transverse Coupling Impedance} beam:

We require the vertical component of the force acting on the

$$
F_{y}=E_{y}+B c B_{x} \text {. }
$$

Since $E_{y}=-\partial V / \partial y$ and $B_{x}=\partial A_{s} / \partial y$, we have

$$
F_{y}=-\left(1-\beta^{2}\right) \frac{\partial V}{\partial y}=-\frac{1}{\gamma} \frac{\partial V}{\partial y} .
$$

The transverse coupling impedance is introduced to provide a simplified force. It is obtained by averaging the coefficient of the rf wave over the circumference. Thus, the transverse impedance $Z_{\perp}$ is

$$
Z_{\perp}=\frac{-i(2 \pi R) F}{\beta T E} y=\frac{i(2 \pi R)}{I E \beta Y^{2}} \frac{\partial V}{\partial y},
$$

with $R$ the average machine radius. 
ISA $78-12$

Introducing

$$
K(z)=\ln \left|\frac{z-z_{1}}{z-b^{2} / z_{1}}\right|-\ln \left|\frac{z-z_{1}^{*}}{z-b^{2} / z_{1}}\right|,
$$

then

$$
z_{\perp}\left(x, x^{\prime}, y\right)=-\frac{i R z o}{2 \beta^{2} \gamma^{2} h} \frac{\partial K}{\partial y} .
$$

Since all field quantities contain the factor $e^{i(k s-w t)}$, we suppress it and simply recall it when it plays a role. we find

Evaluating $z_{\perp}$ at $y=0$, and setting $h=0$ in the image term,

$$
z_{\perp}\left(x, x^{\prime}\right)=\frac{R Z o^{i}}{\beta^{2} \gamma^{2}}\left[\frac{1}{\left(x-x^{\prime}\right)^{2}+h^{2}}-\frac{b^{2}}{\left(b^{2}-x x^{\prime}\right)^{2}}\right] .
$$

To relate this to the "standard" transverse impedance, we must average this over the beam. Consider a small beam of width $t \Delta$, centered at $x=x_{0}$. The "standard" impedance is thus

$$
Z_{\perp}=\frac{1}{2 \Delta} \int_{x_{0}-\Delta}^{x_{0}^{+\Delta}} z_{\perp}\left(x_{0}, x^{\prime}\right) d x^{\prime}
$$

Setting $x^{\prime}=x_{0}$ in the second term, we have

$$
\tilde{Z}_{\perp}=\frac{R Z o^{i}}{\beta^{2} \gamma^{2}}\left[\frac{1}{2 \Delta} \int_{-\Delta}^{\Lambda} \frac{d u}{u^{2}+h^{2}}-\frac{b^{2}}{\left(b^{2}-x_{0}^{2}\right)^{2}} j,\right.
$$

or

$$
\widetilde{Z}_{\perp}=\frac{R_{0} i}{\beta^{2} \gamma^{2}}\left[\frac{1}{\Delta h} \tan ^{-1} \frac{\Delta}{h}-\frac{b^{2}}{\left(b^{2}-x_{0}^{2}\right)^{2}}\right] \text {. }
$$

For a centered "square" beam,

$$
\tilde{Z}_{1}=\frac{-i R z_{0}}{p^{2} \gamma^{2}} \cdots-\frac{\pi}{4 \Delta^{2}}+\frac{1}{b^{2}} \bar{j}
$$

which is essentially the result for a circular beam centered in a circular chamber.

C. Electromagnetic Fields in the Case of Nonperfectly Conducting Walls

We seek a solution for the electromagnetic fields within a conducting medium which has the form of a traveling wave in the direction of the surface. Neglecting the electric displacement current and assuming a conduction current

$$
\mathbf{j}=\sigma E,
$$


with $\sigma$ the conductivity of the medium, then Maxwell's equations within the conducting medium are given by

$$
\begin{array}{ll}
\nabla \times E=i \omega B, & \nabla \cdot E=0, \\
\nabla \times B=\Downarrow \sigma E, & \nabla \cdot B=0 .
\end{array}
$$

A11 the tieids drop immediately to zero in a perfect conductor. Thus, to find an approximate solution in the case of a nonperfect conductor, we make use of the fact that the spatial variation of the fields normal to the surface is much more rapid than the variations parallel to the surface. If $\hat{n}$ is a unit vector normal to the surface, pointing inward, then we can use

$$
\nabla \equiv-\hat{\mathbf{n}} \frac{\partial}{\partial \xi}
$$

where $\xi$ is the coordinate normal to the surface.

From the divergence equations, we have no spatial variation of the normal components of $E$ and $B$. Therefore, within the conductor, we can take approximately

$$
\mathbf{n} \cdot \mathbf{E}=\mathbf{n} \cdot \mathbf{B}=\mathbf{0}
$$

The cur1 equations are

$$
\begin{aligned}
& \frac{\partial}{\partial E}(n \times E)=-i \omega B, \\
& \frac{\partial}{\partial E}(n \times B)=-\mu \sigma E .
\end{aligned}
$$

Let $i$ be a unit vector along the direction of $E$. We write

$$
\mathrm{E}=\mathrm{E}_{\|}(E) \hat{\mathbf{i}} \text {. }
$$

Then, since $n \times n \times E=-E$, we obtain

$$
\frac{d^{2} E_{\|}}{d \xi^{2}}=-i \omega \downarrow \sigma E_{\|}
$$

The skin depth 3 is given by

$$
\delta=\left(\frac{2}{\mu \sigma w}\right)^{i \frac{1}{2}} .
$$

Therefore, the equacion for $\mathrm{E}_{\|}$in a conducting medium is

$$
E_{\|}^{\prime \prime}+\frac{2 i}{\delta} E_{\|}=0 .
$$

The $B$ field is also parallel to the surface, but normal to the $E$ field. Writing $B \equiv B_{\|}$, we have

$$
\mathrm{B}_{\|}=\frac{i}{\omega} \mathrm{F}_{\|}^{\prime},
$$


INA $78-12$

where the direction of $B$ is such that the Poynting vector is inward, meaning that energy is absorbed into the medium.

D. Boundary Conditions at the Surface of a Double Layer Chamber

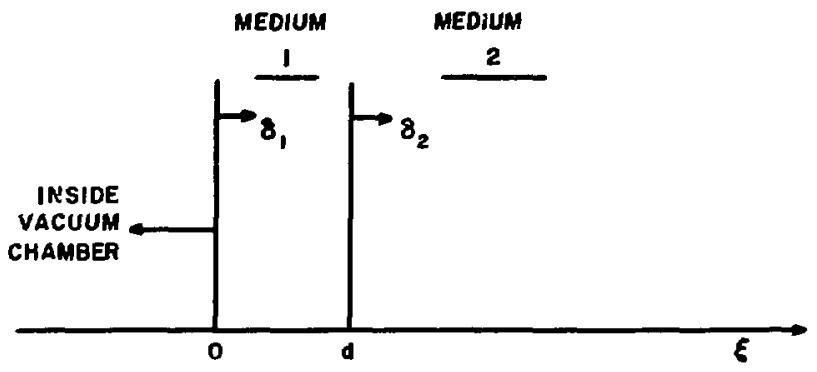

Figure 3. Double layer chamber. $\delta_{1}$ and $\delta_{2}$ are the skin depths for mediums 1 and 2 respectively. d is the thickness of medium 1 . Medium 2 is assumed to have infinite thickness.

We consider the geometry of Figure 3. The equations from (17) and (18) are

$$
E^{\prime \prime}(\xi)+\frac{2 i}{\delta^{2}} E(F)=0,
$$

and

$$
B(\xi)=\frac{i}{\omega} E^{\prime}(F) \text {. }
$$

At the boundary $\xi=\mathrm{d}$, the fields $\mathrm{E}$ and $\mathrm{B}$ are continuous. (This is equivalent to the conditions that the tangential electric and magnetic fields are continuous at the boundaries of real conductors.)

The solution in region 1 is

$$
\begin{aligned}
& E=E_{0} \cosh u_{1} \varepsilon-\frac{i \omega}{u_{1}} B_{0} \sinh \mu_{1} E, \\
& B=\frac{i}{\omega} E^{\prime}=B_{0} \cosh u_{1} E+\frac{i \mu_{1}}{\omega} E_{0} \sinh u_{1} E,
\end{aligned}
$$

where

$$
u_{1}=\frac{\sqrt{2}}{\delta_{1}}(-i)^{\frac{1}{2}}=\frac{1-i}{\delta_{1}} \text {. }
$$

- $8-$ 
At the interface between the chamber wall and the inner space, tine fields are $E_{O}$ and $B_{O}$. What we are seeking is the ratio of electric and magnetic fields just inside the surface - that is, the ratio $E_{o} / B_{o}$. The continuity at the interface tells us that the fields just outside the metallic surface satisfy the same condition. The solution in region 2 is

$$
E=E_{1} e^{-n_{2}(\varepsilon-d)},
$$

which satisfies the condition $E(\xi) \rightarrow 0$ as $\xi \rightarrow \infty$. The constant $u_{2}$ is related to the skin depth in medium $2, \delta_{2}$, by $\varkappa_{2}=(1-i) / \delta_{2}$.

Satisfying the two continuity relations $x t=d$, and eliminating $E_{1}$, leads to

$$
\frac{E_{0}}{i B_{0}}=\frac{w \delta_{1}}{2}(1+i) \frac{\delta_{1}+\delta_{2}-\left(\delta_{1}-\delta_{2}\right) e^{-2 u_{1} d}}{i \delta_{1}+\delta_{2}+\left(\delta_{1}-\delta_{2}\right) e^{-2 u_{1} d}}
$$

E. Fields Resulting from Wall Resistivity

The solution for perfectly conducting boundaries results in an electric field at the boundary which is purely radial and a magnetic field which is purely tangential. Longitudinal fields do not enter in the analysis.

Thus, the effect of including resistance in the walls is to introduce a longitudinal $\mathrm{E}$ field. The tangential $\mathrm{B}$ field must only be continuous at the boundary. If we work only to first order in the resistivity, we can take the tangential magnetic fiald to be unchanged by the wall resistance. Replacing $B_{0} \rightarrow B_{\theta}$ (the tangential component of $B$ with no wall resigtance) and witing $E_{s}$ for the resulting longitudinal $\mathrm{E}$ field, we can obtain $\mathrm{E}_{\mathrm{s}}$ on the boundary from (19):

$$
E_{s}\left(\text { boundary) }=-\frac{(1-i)}{2} \omega \delta B_{\theta}\right. \text {, }
$$

where $\delta$ is a complex effective skin depth,

$$
\delta=\delta_{1}\left[\frac{\delta_{1}+\delta_{2}-\left(\delta_{1}-\delta_{2}\right) e^{-2 x_{1} d}}{-\delta_{1}+\delta_{2}+\left(\delta_{1}-\delta_{2}\right) e^{-2 x_{1} d}} !\right.
$$

Note that the longitudinal $\mathrm{E}$ field is continuous across the chamber surface; thus $E_{s}$ is the value of the field in the vacuum chamber as well as within the metallic surface. To see quantitatively the effect of the two layers, we plot $\left(\delta / \delta_{1}\right)$ in Figure 4 as a function of the two parameters, $\alpha=\delta_{2} / \delta_{1}$ and $\gamma=d / \delta_{1}$. 
ISA $78-12$

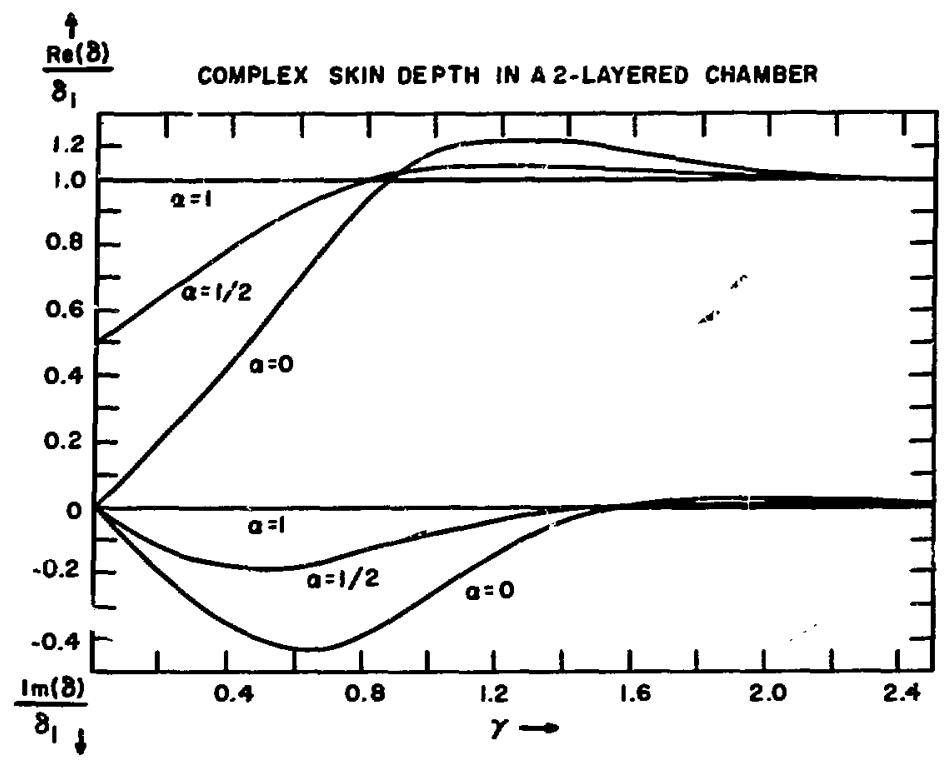

Figure 4. Complex skin depth in a 2 layered chamber. $\alpha=\delta_{2} / \delta_{1}, \gamma=\mathrm{d} / \delta_{1}, \delta_{1}, \delta_{2}$ are skin depths for material 1 (inner layer) and material 2 (outer layer). d is the thickness of the inner layer. The outer layer has infintte thickness.

If we recall that $B=\nabla \times A$, then we have

$$
B_{\theta} \text { (boundary) }=-\frac{\partial A}{\partial r}=-\left.\frac{B}{c} \frac{\partial v}{\partial r}\right|_{r}=b \text {, }
$$

where $V$ is given by (9). Thus

$$
E_{s} \text { (boundary) }=-\left.\frac{(1-i) \omega \delta I Z_{0} \xi e^{i(k s-\omega t)}}{8 \pi c h} \frac{\partial K}{\partial r}\right|_{r=b} \text {. }
$$

We note that the contributions to $K$ from image and self torms are equal except for a constant. For the case here, with two poles, the

constant term vanishes and we can write

$$
G(z)=\frac{\partial K}{\partial r}(z)=\frac{2}{b} \operatorname{Re}\left\{\frac{z}{z-b^{2} / z_{l}}-\frac{z}{z-b^{2} / z_{l}^{*}}\right\}
$$


Now, we are seeking a function $E_{S}(z)$ which satisfies the Laplace equation in the region within $r=b$ and has the value $E_{S}$ (boundary) at $r=b$. Since we have manifestly written (24) as a function of $z$, the function on the right-hand side satisfies the Laplace equation. Thus, we have by analytic continuation of the longitudinal electric field $E_{S}(z)$ everywhere within the chamber:

$$
E_{s}(z)=-\frac{(1-i) \omega \delta I Z_{o} \xi e^{i(k s-\omega t)}}{8 \pi c h} G(z) .
$$

The presence of a longitudinal $\mathrm{E}$ field at the beam introduces a transverse $B$ field and thus a transverse force. From the curl equation $\nabla \times E=-\partial B / \partial t$, we have a horizontal $\mathrm{B}$ field,

$$
B_{x}=\frac{1}{i \omega} \frac{\partial E_{s}}{\partial y} \text {, }
$$

and a vertical force,

$$
F_{y}=B c B=\frac{B C}{i \omega} \frac{\partial E_{s}}{\partial y} \text {, }
$$

or

$$
z_{\perp}\left(x, x^{\prime}, y\right)=\frac{-i R z_{0} \delta(I+i)}{4 h} \frac{\partial G}{\partial y} \text {. }
$$

In the linit $y \rightarrow 0$ and $h+0$, the added impedance resulting from the wall resistivity becomes

$$
z_{\perp}\left(x, x^{\prime}\right)=-i R Z_{0}(1+i) \frac{\delta}{b^{3}} \frac{b^{4}\left(b^{2}+x x^{\prime}\right)}{\left(b^{2}-x x^{\prime}\right)^{3}} .
$$

For a "small" beam, we can set $x=x^{\prime}=x_{0}$ and combine (27) with (15) to find the total impedance,

$\left.\tilde{z}_{\perp}=-i R Z_{0}\left[\frac{1}{\beta^{2} \gamma^{2}} i-\frac{1}{\Delta h} \tan ^{-1} \frac{\Delta}{h}+\frac{b^{2}}{\left(b^{2}-x_{o}^{2}\right)^{2}}\right\}+(1+i) \frac{\delta}{b^{3}} \frac{b^{4}\left(b^{2}+x_{o}^{2}\right)}{\left(b^{2}-x_{o}^{2}\right)^{3}}\right]$.

For a centered beam this becomes

$$
\tilde{Z}_{\perp}=-1 R Z_{0}\left[\frac{1}{\beta^{2} \gamma^{2}}\left\{-\frac{1}{\Delta h} \tan ^{-1} \frac{\Delta}{h}+\frac{1}{b^{2}} j+(1+1) \frac{\delta}{b^{3}}\right]\right. \text {. }
$$

For a wide beam, we combine (14) and (27) to obtain the impedance function

$$
\begin{aligned}
z_{\perp}\left(x, x^{\prime}\right)=-i R z_{\circ} & {\left[\frac{1}{\beta^{2} v^{2}}\left\{-\frac{1}{\left(x-x^{\prime}\right)^{2}+h^{2}}+\frac{b^{2}}{\left(b^{2}-x x^{\prime}\right)^{2}}\right\}\right.} \\
& \left.+(1+i) \frac{\delta}{b^{3}} \frac{b^{4}\left(b^{2}+x x^{\prime}\right)}{\left(b^{2}-x x^{\prime}\right)^{3}}\right] .
\end{aligned}
$$


We can interpret this in terrs of field point and source point. We use the relation between impedance and force,

$$
F_{y}=\frac{i \beta I e \xi}{2 \pi R} Z_{\perp} \text {. }
$$

Therefore, the force at a point $x$ due to a source at $x^{\prime}$ is

$$
F_{y}\left(x, x^{\prime}\right)=\frac{i \beta I e}{2 \pi R} z_{\perp}\left(x, x^{\prime}\right) \xi\left(x^{\prime}\right),
$$

with $z_{\perp}$ given by $(30)$ and the phase factor $e^{i(k s \cdot j t)}$ implied.

F. Lorentz Force and Integral Equation for the Perturbed Amplitude

The motion of a particle of momentum $p$ in a force field $F$ is

$$
\frac{d p}{d t}=F(\text { total) }
$$

If $\mathrm{y}$ is the vertical displacement of a particle of rest mass $\mathrm{m}$, then the transverse momentum is

$$
\mathrm{P}_{\mathrm{y}}=\mathrm{m} \vee \dot{\mathrm{y}} \text {. }
$$

Separating out from $F$ (total) the focusing field due to the magnetic structure around the ring, we find that the transverse betatron motion of a particle is described by the perturbed harmonic oscillator equation

$$
\ddot{\mathrm{y}}+\left(v \omega_{\mathrm{o}}\right)^{2} \mathrm{y}=\mathrm{F}_{\mathrm{y}} / \mathrm{m} v,
$$

where $v$ is the particle tune and $F_{y}$ is the force due to the beam induced fields we have been discussing.

We are looking for a solution where all the particles travel along the tube in a wave of the form $e^{i(k s-b t)}$. To obtain the acceleration of a particle, we must travel with it. Thus, the time dependence is obtained by setting $s \rightarrow R \omega_{0} t$. Introducing the mode number $n$,

$$
\mathbf{n}=\mathbf{k} \mathbf{R},
$$

the phase factor takes the form $e^{i\left(n w_{0}-w\right) t}$.

Therefore, the time derivative in (32), for the collective solutions being sought, can be replaced by $i\left(n \omega_{0}^{-(\omega)}\right)$ : that is,

$$
\frac{d}{d t} \rightarrow i\left(n w_{0}-w\right)
$$

We have then

$$
\left[-\left(n \omega_{0}-w\right)^{2}+\left(v w_{0}\right)^{2}\right] y=F_{y} / m v \text {. }
$$

We can now omit the phase factor since it appears on both sides of (34) and cancels. 
Consider an ensemble of $\mathrm{N}$ particles $\mathrm{y}_{i}$ in a strip of height $2 h$ and sinall (infinitesimal) width at the horizontal position $x$. The mean displacement of these particles is simply the amplitude $E$ defined in $(1)$ :

$$
\frac{1}{N} \sum_{i=1}^{N} y_{i}=\xi \text {. }
$$

We assume that $F_{y}$ is slowly varying in $y$ and simply evaluate it at $y=0$. Further, we take $v$ to be $y$ independent. Therefore, averaging (34), we obtain

$$
\left[-\left(n \omega_{0}-w\right)^{2}+\left(v \omega_{0}\right)^{2} i \xi(x)=F_{y} /\left.m y\right|_{y=0} .\right.
$$

Now, the force on a given strip at the point $x$ has contributions from the complete beam. The particular contribution from a source at $x^{\prime}$ has been written in the corm [Eq. (31)]

$$
F_{y}\left(x, x^{\prime}\right)=\frac{i \beta I e}{2 \pi R} z_{\perp}\left(x, x^{\prime}\right) \xi\left(x^{\prime}\right) \text {. }
$$

This is, in other words, the force at a point $x$ due to a source at $x^{\prime}$ with $E\left(x^{\prime}\right)$ the vertical coherent displacement in the beam at the source point, $x^{\prime}$. The total force is obtained by summing over the source "strips". Introducing a density function $o(x)$ normalized to unity,

$$
\int_{\substack{\text { Beam } \\ \text { Width }}} \rho(x) d x=1 \text {, }
$$

(36) can be written

$$
\left\{-\left(n \omega_{0}-w\right)^{2}+\left[v(x) \omega_{0}\right]^{2}\right\} \xi(x)=\frac{F_{y}(x)}{m y},
$$

where

$$
F_{y}(x)=\left.\int F_{y}\left(x, x^{\prime}\right) \rho\left(x^{\prime}\right) d x^{\prime}\right|_{y=0},
$$

and we have explicitly noted that the betatron tune, $v$, is considered a function of the beam position, $x$.

We can simplify the equation (38) by noting that the two solutions for $\omega$ correspond to fast and slow waves, $\omega \approx(n+\nu) \psi_{0}$ and $\omega \approx(n-\nu) \omega_{0}$ respectively. Although the fast or "backward" traveling wave is a possible solution, it is one that does not lead to growing coherent oscillations, but rather to damped oscillations. Since over a long time such waves do not persist, we can simply ignore this mode. Thus, we can simplify (38) and write it in the form,

$$
\left[v(x)-\left(n-\frac{w}{w_{0}}\right) j \xi(x)=\frac{F(x)}{2 m \vee w_{0}^{2} v_{0}},\right.
$$


where $v_{a}$ is the betatron tune at the beam center. Inserting (31) and using the expression for the classical proton radius,

$$
r_{p}=\frac{e^{2}}{4 \pi \epsilon_{o} m c^{2}}=\frac{e^{2 z_{o}}}{4 \pi m c},
$$

we obtain

$$
\left[\nu(x)-\left(n-\frac{\omega}{\omega_{0}}\right)\right] \xi(x)=i\left(\frac{I}{e}\right) \frac{{ }^{x} p}{v \omega_{0} v_{0} z_{0}} \int z_{\perp}\left(x, x^{\prime}\right) \rho\left(x^{\prime}\right) \xi\left(x^{\prime}\right) d x^{\prime},
$$

which is a homogenecus integral equation for the perturbed coherent amplitude, $\xi(x)$, with the transverse impedance function $z_{\perp}\left(x, x^{\prime}\right)$ acting as a sort of Green's function, providing the internal coupling for the system of oscillators.

G. Model with a Linear Correspondence of Tune and Horizontal Position

The equation for the perturbed amplitude can be simplified by invoking a correspondence between tune and chamber location. This does, in fact, correspond to realicy in that the operating machine chromaticity does indeed lead to such a relationship. The connection is norma11y linear and we will make this assumption. by

Let the beam have its center at $x=x_{0}$ and a finite range given

$$
\text { Range }\left[x-x_{0}\right]= \pm \Delta \text {. }
$$

In tune space, we take for the range

$$
\text { Range }\left[v-\nu_{0}\right]= \pm d,
$$

with $v_{0}$ the beam tune center corresponding to the beam position, $x_{0}$. Taking a linear relation between $v$ and $x$, we have

$$
v(x)=v_{0}+\frac{d}{\Delta}\left(x-x_{0}\right) \text {. }
$$

Inserting $x$ in place of $v$ in $(40)$, we can write

$$
\left(x-x_{c}\right) \xi(x)=\int G\left(x, x^{\prime}\right) \rho\left(x^{\prime}\right) \xi\left(x^{\prime}\right) d x^{\prime},
$$

where the coherent frequency in $x$ is

$$
x_{c}=x_{0}-\frac{\Delta}{d}\left(v_{0}-n+\frac{w}{\omega_{0}}\right),
$$

and where the oscillator coupling function or effective Green's function is given by

$$
G\left(x, x^{\prime}\right)=i\left(\frac{I}{e}\right) \frac{r_{p} \Delta}{\gamma y_{0}^{\prime} v_{0} z_{0}^{d}} z_{\perp}\left(x, x^{\prime}\right) .
$$


We can perform one more simplification by using a nomalized space variable, $u$, defined by

$$
u=\left(x-x_{0}\right) / \Delta \text {. }
$$

The variable $u$ thus defines the extent of the beam in space and has the value 0 at the beam center and the range,

$$
\text { Range }[u]= \pm 1 \text {. }
$$

If we rodefine the density to be normalized to unity with respect to $u$, that is,

$$
\int_{-1}^{1} \rho(u) d u=1
$$

then (42) can be expressed by

$$
\left(u-u_{c}\right) \xi(u)=\int G\left(u, u^{\prime}\right) \rho\left(u^{\prime}\right) \xi\left(u^{\prime}\right) d u^{\prime},
$$

where the coherent frequency $u_{c}$ is

$$
u_{c}=\frac{1}{d}\left(n-v_{0}-\frac{\omega}{\omega_{o}}\right),
$$

and the coupling function is redefined to be

$$
G\left(u, u^{\prime}\right)=i\left(\frac{I}{e d}\right) \frac{r_{p}}{y w_{0} v_{0} z_{0}} z_{\perp}\left(u, u^{\prime}\right),
$$

with $z_{\perp}\left(u, u^{\prime}\right)$ the same function as $z_{\perp}\left(x, x^{\prime}\right)$ with $x$ and $x^{\prime}$ replaced by $u$ and $u^{\prime}$ via (45).

H. Dymamics in the Case of a Sma11 Beam

We start with the integral equation for $\xi$ :

$$
\left(u-u_{c}\right) \xi(u)=\int G\left(u, u^{\prime}\right) \rho\left(u^{\prime}\right) \xi\left(u^{\prime}\right) d u^{\prime} .
$$

If the beam is small we can approximate $G\left(u, u^{\prime}\right)$ by its value at the beam center, except that the beam term is averaged as in section $2 B$. Thus, from (49), and using the "sma11" beam impedance function, Eq. (28), we obtain

$$
\begin{aligned}
G\left(u, u^{\prime}\right) \equiv \widetilde{G}=\left(\frac{I}{e d}\right) \frac{r_{p} R}{\gamma w_{0} v_{0}} & \left\{\frac{1}{\beta^{2} \gamma^{2}}\left[-\frac{1}{\Delta h} \tan ^{-1} \frac{\Delta}{h}+\frac{1}{b^{2}} \frac{1}{\left(1-t_{0}^{2}\right)}\right]\right. \\
& \left.+(1+i) \frac{\delta}{b^{3}} \frac{\left(1+t_{0}^{2}\right)}{\left(1-t_{0}^{2}\right)^{3}}\right\},
\end{aligned}
$$


where we have introduced $t$, the ratio of space position to chamber radius, in place of $x$. In particular, the beam center is desigiated by

$$
t_{0}=x_{0} / b
$$

Thus, the integral equation becomes

$$
\left(u-u_{c}\right) \varepsilon(u)=\widetilde{G} \int \rho\left(u^{\prime}\right) \varepsilon\left(u^{\prime}\right) d u^{\prime} .
$$

We recall that the phase factor $e^{-i \text { ut }}$ describes the time evolution of the beam amplitude as well as the induced fields. Since we are discussing a physical situation which is assumed to have existed for a long time and which will continue in the future, solutions that damp are not admissible. In other words, we are looking for solutions with $\operatorname{Im}(\omega) \geq 0$. The quantity $\operatorname{Im}(\omega)$, when it exists, is just the e-folding growth rate,

$$
\operatorname{Im}(\omega)=1 / \tau \text {. }
$$

By a threshold solution is meant the limit of zero growth rate, or $\operatorname{Im}(\omega)+0^{+}$. By (48), $u_{c}$ and $w$ differ in sign. In fact,

$$
\operatorname{Im}(w)=-w_{0} d \operatorname{Im}\left(u_{c}\right) \text {. }
$$

Thus, a threshold solution is found by takint the limit,

$$
\operatorname{Im}\left(\mathbf{u}_{\mathbf{c}}\right) \rightarrow 0^{-} \text {. }
$$

Under unstable conditions the mean observed signal is simply

$$
S=\int \rho\left(u^{\prime}\right) \xi\left(u^{\prime}\right) d u^{\prime}
$$

Thus, Eq. (51) becomes

$$
\xi(u)=\frac{\widetilde{G} s}{u-u_{c}} .
$$

Multiplying by $\rho(u)$ awi integrating, we obtain

$$
s\left[1-\widetilde{G} \int \frac{\rho(u)}{u-u_{c}} d u\right]=0 .
$$

Therefore, for a nonzero signal to survive, we must satisfy the condition on the coherent frequency $\mathbf{u}_{c}$,

$$
1=\tilde{G} \int \frac{\rho(u)}{u-u_{c}} d u .
$$

For a sustained but nongrowing signa1, that is, for a threshold, we must define passage through the singular point $u u_{c}$ by taking the limit, $\operatorname{Im}\left(u_{c}\right) \rightarrow 0^{-}$. 
To estimate the growth rate, we take a de:1t 1 function density distribution,

$$
p(\mathbf{u})=\delta(\mathbf{u}),
$$

which, from (57), leads to

$$
u_{c}=-\widetilde{G} \text {. }
$$

Recalling that the growth rate is

$$
\frac{1}{\tau}=\operatorname{Im}(\omega)=-\omega_{0} d \operatorname{Im}\left(u_{c}\right),
$$

we have that the growth rate far from threshold is roughly given by

$$
\frac{1}{T}=\omega_{0} \mathrm{~d} \operatorname{Im}(\widetilde{\mathrm{G}})
$$

To see how the dispersi i relation is to be dealt with in finding the current threshold, take a uniform density distribution,

$$
\rho(u)=\frac{1}{2},-1 \leq u \leq 1 \text {. }
$$

Equation (57) becomes

$$
\frac{2}{\tilde{G}}=\int_{-1}^{1} \frac{d u}{u-u_{c}} .
$$

To find the threshold, take $u_{c}$ to have a small, negative imaginary part,

$$
u_{c}=u_{R}-i \epsilon \text {. }
$$

Taking the limit $\epsilon \rightarrow 0$, we obtain for the threshold equation,

$$
\frac{2}{\tilde{G}}=P \int_{-1}^{1} \frac{\mathrm{du}}{\mathrm{u}-\mathrm{u}_{\mathrm{R}}}-\mathrm{i} \pi \text {, for }-1<\mathrm{u}_{\mathrm{R}}<1 \text {, }
$$

where $P$ stands for Principal Value Integral. Thus, we have for the threshold condition

$$
\frac{2}{\tilde{G}}=\ln \left|\frac{1-u_{R}}{1+u_{R}}\right|-i \pi,-1<u_{R}<+1 \text {. }
$$


Setting the real part of $(60)$ to zero gives the real osciliation frequency, $u_{R}$, while taking the imaginary part of both sides leads to the current threshold. With

$$
\widetilde{G}=\widetilde{G}_{R}+i \widetilde{G}_{I},
$$

the threshold current ia found immediately from

$$
\frac{\widetilde{G}_{I}}{\tilde{G}_{R}^{2}+\widetilde{G}_{I}^{2}}=\pi / 2
$$

while the real frequency shift, $u_{R}$, is obtained from

$$
\frac{\widetilde{G}_{R}}{\widetilde{G}_{R}^{2}+\tilde{G}_{I}^{2}}=\frac{1}{2} \ln \left|\frac{1-u_{R}}{1+u_{R}}\right| \text {, }
$$

where the current used in (62) is the threshold value found from (61).

In the case of a more general density distribution function, $p(u)$, the threshold current and frequency shift are found from the complex equation

$$
\frac{2}{\widetilde{G}}=\mathrm{P} \int_{-1}^{1} \frac{\rho(u)}{u-u_{R}} d u \quad-i \pi \rho\left(u_{R}\right) ; \quad-1<u_{R}<1 .
$$

I. Solution of the Integra1 Equation for a Wide Beam by Interpolation density,

The Green's function $G\left(u, u^{\prime}\right)$ is proportional to the tune current

$$
\mathbf{i}=\mathrm{I} / 2 \mathrm{~d},
$$

the ratio of the beam current to the total ture spread. If we normalize to a particular current density, $i_{0}$, then we can define $G$ with respect to this normal current density and introduce a parameter $\eta$ to describe deviations from the normal value. Thus, we replace

$$
G(i) \rightarrow \eta G\left(i_{0}\right),
$$

and the integral equation takes the form,

$$
\left(u-u_{c}\right) E(u)=\eta \int G\left(u, u^{\prime}\right) \rho\left(u^{\prime}\right) E\left(u^{\prime}\right) d u^{\prime} .
$$

As we will see, it is convenient to view (65) as an integral equation for the eigenvalue $\eta$ with corresponding eigenvector $\xi(u)$, given the complex frequency, $u_{c}$.

As is clear from (56), we expect $\xi(u)$ to be a rapidly varying function for $u$ near $u_{c}$, which will be the case near threshold. We, therefore, in roduce a new function, $f(u)$, which is a slowly varying 
function across $u=u_{c}$ :

$$
f(u)=\left(u-u_{c}\right) \xi(u) \rho(u) \text {. }
$$

This transforms the integral equation (65) into the equation

$$
f(u)=\eta \rho(u) \int_{-1}^{1} \frac{G\left(u_{,} u^{\prime}\right) f\left(u^{\prime}\right)}{u^{\prime}-u_{c}} d u^{\prime},
$$

where the range of integration has been nornalized to $[-1,1]$. A11 the functions are weak functions of $u$ and $u^{\prime}$ in the range of interest. It is, therefore, clear that the key to solving (67) hinges on how the singularity at $u^{\prime}=u_{c}$ is dealt with.

Splitting the integrand into singular and nonsingular parts by writing

$$
G\left(u, u^{\prime}\right) \pm G\left(u, u_{c}\right)+\left[G\left(u_{:^{\prime} \prime^{\prime}}\right)-G\left(u, u_{c^{\prime}}\right]\right. \text {, }
$$

the integral equation (67) takes the form

$f(u)=\eta p(u)\left\{G\left(u, u_{c}\right) \int_{-1}^{\prime 1} \frac{f\left(u^{\prime}\right)}{u^{\prime}-u_{c}} d u^{\prime}+\int_{-1}^{1} \frac{G\left(u, u^{\prime}\right)-G\left(u_{,} u_{c}\right)}{u^{\prime}-u_{c}} f\left(u^{\prime}\right) d u^{\prime}\right\}$,

where $G\left(u, u_{c}\right)$ is obtained by analytic continuation and the integrand in the second term on the right-hand side is well behaved as $u^{\prime}$ passes through the singular point $u_{c}$.

We evaluate the integrals by an extension of simpson's rule. Divide the to al u range, $[-1,1]$, into an even number of equal intervals, $2 M$. This requires an odd number of points, $2 M+1$, designated as $u_{1}=-1, u_{2}, u_{3}, \ldots \ldots . u_{2 M}, u_{2 M+1}=1$. The length of each of the equal intervals is

$$
\mathrm{h}=1 / \mathrm{M} \text {. }
$$

The second integral in (68) is regular and can be evaluated by a direct application of Simpson's rule. We write

$$
H\left(u, u^{\prime}\right)=\frac{G\left(u, u^{\prime}\right)-G\left(u, u_{c}\right)}{u^{\prime}-u_{c}} \text {. }
$$


In the interval from $u_{n-1}$ to $u_{n+1}$, witin $n$ taken to be even, we have

$$
\begin{aligned}
& \int_{u_{n-1}}^{u} H\left(u, u^{\prime}\right) f\left(u^{\prime}\right) d u^{\prime} \\
& =\frac{h}{3}\left[H_{n-1} f_{n-1}+4 H_{n} f_{n}+H_{n+1} f_{n+1}\right],
\end{aligned}
$$

where $f_{n-1}, f_{n}$, and $f_{n+1}$ are the values of $f(u)$ at $u=u_{n-1}, u_{n}$ and $u_{n+1}$ respectively, with similar meanings for $H_{n-1}, H_{n}$, and $H_{n+1}$, ihus the total integral is given by

$\int_{-1}^{1} H\left(u, u^{\prime}\right) f\left(u^{\prime}\right) d u^{\prime}=\frac{h}{3} \sum_{\text {even }}\left[H_{n-1} f_{n-1}+4 H_{n} f_{n}+H_{n+1} f_{n+1}\right]$.

To Evaluate the "singular" integral, we use an extended Simpson's rule, approximating $f\left(u^{\prime}\right)$ by a parabola in each interval $u_{n-1} \leq u^{\prime} \leq u_{n+1}$, leaving alone the "singular" factor, $1 /\left(u^{\prime}-u_{c}\right)$. In particular, $f\left(u^{\prime}\right)$ is approximated by a parabola passing through the three points $u_{n-1}, u_{n}$, and $u_{n+1}$ :

$$
\begin{aligned}
f(u) & =A_{n} u^{2}+B_{n} u+C_{n}, \\
A_{n} & =\frac{1}{2 h^{2}}\left[f_{n-1}-2 f_{n}+f_{n+1}\right], \\
B_{n} & =\frac{1}{2 h}\left[f_{n+1}-f_{n-1}\right]-2 u_{n} A_{n}, \\
C_{n} & =f_{n}-\frac{u_{n}}{2 h}\left[f_{n+1}-f_{n-1}\right]+u_{n} A_{n} .
\end{aligned}
$$

Performing the integration in the interval $\left[u_{n-1}, u_{n+1}\right]$, we have

$$
\begin{aligned}
\int_{u_{n-1}}^{u} \frac{f\left(u^{\prime}\right)}{u^{\prime}-u_{c}} d u^{\prime}= & -\left(1+\frac{1}{2} \Delta_{n} L_{n}\right)\left(1-\Delta_{n}\right) f_{n-1} \\
& +\left[\left(1-\Delta_{n}^{2}\right) L_{n}-2 \Delta_{n}\right] f_{n} \\
& +\left(1+\frac{1}{2} \Delta_{n} L_{n}\right)\left(1+\Delta_{n}\right) f_{n+1},
\end{aligned}
$$


where

$$
\Delta_{n}=\left(u_{c}-u_{n}\right) / h \text {, }
$$

and the singular nature of the integral is contained in the term, $L_{n}$, given by

$$
L_{n}=\int_{u_{n-1}}^{u_{n+1}} \frac{d u^{\prime}}{u^{\prime}-u_{c}}
$$

Writing

$$
u_{c}=u_{R}+i u_{I},
$$

we perform the integral in (74), leading to

$L_{n}=\frac{3}{2} \ln \left\{\frac{\left(u_{n+1}-u_{R}\right)^{2}+u_{I}^{2}}{\left(u_{R}-u_{n-1}\right)^{2}+u_{I}^{2}}\right\}+i\left\{\tan ^{-1}\left(\frac{u_{n+1}-u_{R}}{u_{I}}\right)+\tan ^{-1}\left(\frac{u_{R}-u_{n-1}}{u_{I}}\right)\right\}$,

where the function $\tan ^{-1}$ is defined in the range $[-\pi / 2,+\pi / 2]$. Our analysis is applicable for solutions with $u_{I} \leq 0$. Such solutions correspond to steady state $\left(u_{I}=0\right)$ or growing $\left(u_{I}<0\right)$ coherent beam oscillations. For the threshold solution, we take the limit $u_{I} \rightarrow 0^{-}$. Giving $u_{c}$ a snali negative imaginary part and taking the limit, we obtain

$$
I_{n}=\begin{array}{ll}
\ln \left|\frac{u_{n+1}-u_{R}}{u_{R}^{-u_{n-1}}}\right|-i \pi & u_{n-1}<u_{R}<u_{n+1} \\
\ln \left|\frac{u_{n+1}-u_{R}}{u_{R}^{-u_{n-1}}}\right| & u_{n+1}<u_{R}, u_{n-1}>u_{R}
\end{array}
$$

From (72), we find for the total "singular" integra1,

$$
\begin{aligned}
\int_{-1}^{1} \frac{f\left(u^{\prime}\right)}{u^{\prime}-u_{c}} d u^{\prime}= & \sum_{\substack{\text { even } \\
n}}\left[-\left(1+\frac{1}{2} \Delta_{n} L_{n}\right)\left(1-\Delta_{n}\right) f_{n-1}+\left[\left(1-\Delta_{n}{ }^{2}\right) L_{n}-2 \Delta_{n}\right] f_{n}\right. \\
& \left.+\left(1+\frac{\pi}{2} \Delta_{n} L_{n}\right)\left(1+\Delta_{n}\right) f_{n+1}\right],
\end{aligned}
$$

where $L_{n}$ is given by (76) or (77) in the threshold case, $u_{I} \rightarrow 0^{-}$, while $\Delta_{n}^{n}$ is given by (73) or in the threshold case,

$$
\Delta_{n}=\left(u_{R}-u_{n}\right) / h
$$


The procedure given here converts the integral equation (67) into the algebraic equation

$$
\mathrm{f}_{\ell}=\eta \sum_{\mathrm{m}=1}^{2 \mathrm{M}+1} \mathrm{~T}_{\ell: \pi} \mathrm{f}_{\mathrm{m}} .
$$

Combining the above results, we can express the $T$ matix as

$$
\mathrm{T}_{\ell \mathrm{m}}=\rho_{\ell} \mathrm{G}_{\ell} \mathrm{R}_{\mathrm{m}}+(1 / 3) \mathrm{h} \mathrm{S}_{\ell, \mathrm{m}} \text {, }
$$

where

$$
\rho_{\ell}=\rho\left(u_{l}\right) \text {, }
$$

and

$$
\mathbf{G}_{\ell}=\mathbf{G}\left(\mathbf{u}_{\ell}, \mathbf{u}_{\mathbf{c}}\right) \text {. }
$$

The quantities $\mathrm{R}_{\mathrm{m}}$ and $\mathrm{S} \ell, \mathrm{m}$ are somewhat more complicated. The quantity $R_{m}$ is given as foìlows:

$$
\begin{aligned}
& R_{m}=\left(1-\Delta_{m}^{2}\right) L_{m}-2 \Delta_{m} \quad \text {, for even } m=2,4, \ldots 2 M ; \\
& R_{1}=-\left(1+\frac{1}{2} \Delta_{2} L_{2}\right)\left(1-\Delta_{2}\right) \text {, for odd } m=1 \text {; } \\
& R_{m}=-\left(i+\frac{1}{2} \Delta_{m+1} L_{m+1}\right)\left(1-\Delta_{m+1}\right) \\
& +\left(1+\frac{2}{2} \Delta_{m-1} L_{m-1}\right)\left(1+\Delta_{m-1}\right) \text {, for odd } m=3,5 \ldots 2 m-1 \text {; } \\
& R_{2 M+1}=\left(1+\frac{1}{2} \Delta_{2 M} L_{2 M}\right)\left(1+\Delta_{2 M}\right) \text {, for ord } m=2 M+1 \text {. }
\end{aligned}
$$

The matrix $s_{\ell, m}$ is given as follows:

$$
\begin{aligned}
s_{\ell, m} & =4 H_{\ell, m}, \text { for even } \mathrm{m}=2,4 \ldots 2 \mathrm{M} ; \\
s_{\ell, 1} & =\mathrm{H}_{\ell, 1}, \text { for odd } \mathrm{m}=1 ; \\
\mathrm{s}_{\ell, \mathrm{m}} & =2 \mathrm{H}_{\ell, \mathrm{m}}, \text { for od } \mathrm{m}=3,5 \ldots 2 \mathrm{M}-1 ; \\
\mathrm{s}_{\ell,(2 \mathrm{M}+1)} & =\mathrm{H}_{\ell,(2 M+1)}, \text { for odd } \mathrm{m}=2 \mathrm{M}+1 ;
\end{aligned}
$$

with the matrix $\mathrm{H}_{\ell, \mathrm{m}}$ given by

$$
H_{\ell, m}=\frac{G\left(u_{\ell}, u_{m}\right)-G\left(u_{\ell}, u_{c}\right)}{u_{m}-u_{c}} .
$$




\section{J. The Eigenvalue Problen for the Current Density}

We have introduced a normalized current density $\eta$. We have shown that the coherent motion can be expressed in terms of the algebraic equation

$$
(T-I / T) \mathbf{f}=0
$$

where $T$ is the matrix defined by (81), I is the unit matrix, and $f$ is the vector amplitude. The actual coherent beam amplitude function $\xi$ is related to $f$ by (66).

Equation (85) is in the form of a simple eigenvalue problem in terms of the parameter $\eta$. However, given any value of $u_{c}$, the eigenvalue $\eta$ is in general complex. On the other hand, a physically meaningful solution must have $\eta$ reai, since it represents a real current.

In practice, (35) is solved by first choosing a value for $u_{I}$. In the case of the thrashold problem, we take $u_{I} \rightarrow 0^{-}$. Then (85) is solved for comflex $\eta$ for different values of $u_{P_{0}}$ A physical solution is obtained for that value of $u_{R}$ giving $\operatorname{Im}(\eta)=0$. The current corresponding to the given value of growth rate $u_{I}$ is just given by $\operatorname{Re}(\eta)$, and the frequency shift is the obtained value of $u_{R}$. In particular, the problem of finding the current threshold $\eta^{R}$ is reduced to the problem of finding the value of $u_{R}$ which produces a real eigenvalue $\eta$, having taken $u_{I} \rightarrow 0^{-}$. In the case of finding the threshold, a further condition results from the fact that the threshold is a limiting value of $u_{I}=0$ with $u_{I}$ constrained to be increaving as the current tecreases toward threshold. Thus, if $u_{I}$ is considered a function of the current $\eta$, the limit $u_{I} \rightarrow 0^{-}$means: $u_{I}=0, u_{I}^{\prime}=d u_{I} / d \eta<0$.

To find the threshold current, instead of looking at $u_{I}$ as a function of the physical current, $\eta$, we look at $\operatorname{Im}(\eta)=\eta_{I}$ as a function of $u_{R}$. Now, in general, the complex variable $\eta$ is a function of the complex variable $u_{c}$, with

$$
\frac{d \eta}{d u_{c}}=\left(\frac{d u_{c}}{d \eta}\right)^{-1} \text {. }
$$

Taking $\eta_{I} \rightarrow 0$ and $u_{I} \rightarrow 0$, we have

$$
\left.\frac{d \eta}{d u_{R}}\right|_{u_{I}=0}=\left.\left(\frac{d u_{R}}{d \eta_{R}}+i \frac{d u_{I}}{d \eta_{R}}\right)^{-1}\right|_{\eta_{I=0}},
$$

or, taking imaginary parts,

$$
\frac{d \eta_{I}}{d u_{R}}=-\frac{u_{I}^{\prime}}{\left(u_{R}^{\prime 2}+u_{I}^{\prime 2}\right)}
$$

Since the actual ptysical solution demands $u_{I}^{\prime}<0$, we must impose the condition $d \eta_{I} / d u_{R}>0$ on the solution we obtain for the threshold.

In looking for solutions for the threshold current, we find that for a wide beam, there may exist more than one coherent mode. These correspond to different frequencies, which means that the different modes 
are governed by oscillations manifesting themselves in different sectors of the wide beam. Mathematical1y, this means that the curve $\eta_{I}\left(u_{R}\right)$ passes through zero more than once. What the canstraint (88) means is that those candidates for a solution, that is, $\eta_{I}\left(u_{R}\right)=0$, for which $d \eta_{I} / d u_{R}<0$, must be discarded as nonphysical. Ion the other hand, those with $\eta_{I}\left(u_{R}\right)=0$ and $d \eta_{I} / d u_{R}>0$ represent physically realizable coherent modes.

\section{RESULTS WITH MODELS FOR THE ISABELLE BEAM}

\section{A. ISABELLE Parameters}

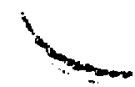

The general ISABELLE parameters common to all the models we will use are listed in Table $I$, while those beam parameters common to all models are given in Table II.

\section{B. Coherent Instability with a Point Beam Mode1}

In the case of a small beam, we use the theoretical model developed in Section $2 \mathrm{H}$. In determining the threshold, we use both a uniform tune distribution and a flat distribution with $\sin ^{2}$ tails on each side. Case $I$ is therefore:

$$
\rho(u)=\frac{1}{2},-1 \leq u \leq 1 \text {; }
$$

while Case II has the distribution as follows:

$$
\begin{gathered}
\quad c \sin ^{2} \frac{\pi}{2}\left(\frac{1+u}{1-\alpha_{L}}\right)-1 \leq u \leq-\alpha_{I_{1}}, \\
\quad c \sin ^{2} \frac{\pi}{2}\left(\frac{1-u}{1-\alpha_{R}}\right) \quad-\alpha_{L} \leq u \leq \alpha_{R},
\end{gathered}
$$

wi th

$$
c=1 /\left[1+\frac{1}{2}\left(\alpha_{L}+\alpha_{R}\right)\right] \text {. }
$$

To see the effect of tails, we choose the distribution with $20 \%$ tails and $80 \%$ flat. That is, we take $\alpha_{\mathrm{L}}=\alpha_{\mathrm{R}}=0.8$.

For a "small" beam, we take a beam which contains $20 \%$ of the nominal full ISABELLE current. This could represent the first in a sequence of stacking steps during the current accumulation process. The beam parameters other than those already described are given in Table III. 
Table I: General ISABELLE Parameters

\begin{tabular}{|c|c|c|}
\hline Parameter & Vali:e & Expression or comment \\
\hline Average radius, $R$ & $599.5 \mathrm{~m}$ & $\mathrm{C}=42 / 3 \mathrm{C}_{\mathrm{AGS}}$ \\
\hline Central tune, $\nu_{0}$ & 22.62 & \\
\hline Revolution frequency, $f_{0}$ & $79.64 \mathrm{kHz}$ & $f_{0}=\beta c / 2 \pi R$ \\
\hline Beam energy, $y$ & 31.3 & $y=E / m c^{2}(E=29.4 \mathrm{GeV})$ \\
\hline Chamber radius, b & $4.4 \mathrm{~cm}$ & \\
\hline Resistivity, ss, $\rho_{1}$ & $1.0 \times 10^{-6} \Omega \mathrm{m}$ & \\
\hline $\begin{array}{l}\text { Resistivity, } \mathrm{Cu}, \mathrm{P}_{2} \\
\text { Thickness, ss layer, d }\end{array}$ & $\begin{array}{r}1.7 \times 10^{-8} \mathrm{sm} \\
1.0 \mathrm{~mm}\end{array}$ & Layer outside ss chanber \\
\hline Azimuthal mode, $\mathrm{n}$ & 23 & $\mathrm{n}=24,25 \ldots \ldots$ also possible \\
\hline $\begin{array}{l}\text { Resonant frequency for } \\
\text { mode } n, f_{r}\end{array}$ & $30.26 \mathrm{kHz}$ & $\mathbf{f}_{\mathbf{r}}=\left(n-v_{0}\right) f_{0}(n=23)$ \\
\hline Skin depth, ss, $\delta_{1}$ & $2.89 \mathrm{~mm}$ & $\delta=(p c / \pi z \underset{f}{f})^{\frac{1}{2}}(n=23)$ \\
\hline $\begin{array}{l}\text { Skin depth, } \mathrm{Cu},{ }_{2}{ }_{2} \\
\text { Thickness, Cu layer }\end{array}$ & $\begin{array}{c}0.38 \mathrm{~mm} \\
\infty\end{array}$ & Effectively, thickness $\gg \diamond_{2}$ \\
\hline $\begin{array}{l}\text { Complex effective skin } \\
\text { depth, } 8\end{array}$ & $(1.414-0.8161) \mathrm{mm}$ & Eq. (21) $(n=23)$ \\
\hline
\end{tabular}

Table II: ISABELLE Beam Parameters

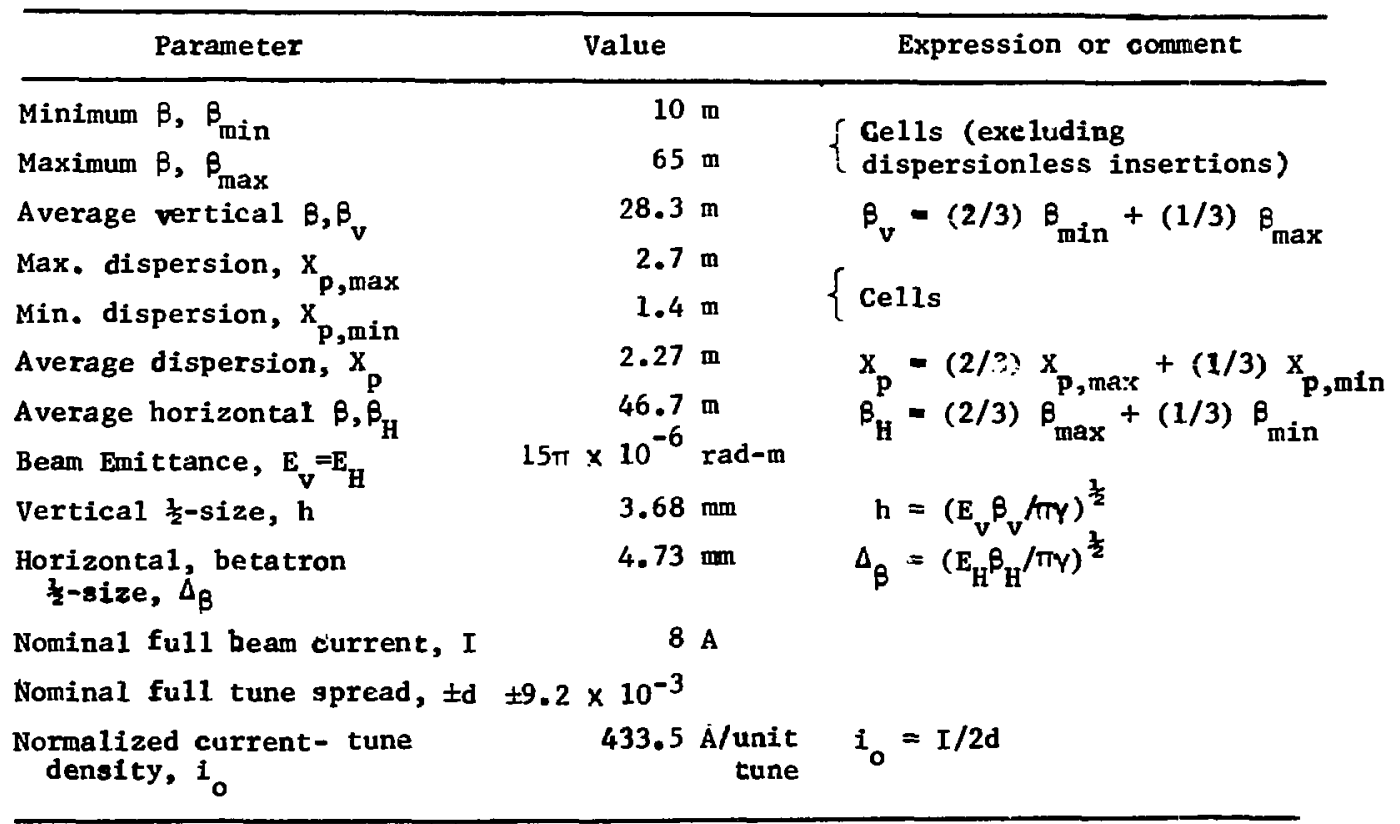


Table III: Beam Parameters with Point Beam Mode1

\begin{tabular}{lll}
\hline \multicolumn{1}{c}{ Parameter } & \multicolumn{1}{c}{ Value } & Expression or comment \\
\hline Beam surrent, $I$ & $1.6 \mathrm{~A}$ & $20 \%$ of Nominal full current \\
Tune spread, $\pm d$ & $\pm 1.84 \times 10^{-3}$ & $20 \%$ of Nominal spread \\
Momentum spread, $\pm \sigma_{p}$ & $\pm 0.1 \%$ & $20 \%$ of Nominal stack spread \\
Momentum $\frac{1}{2}$ size, $\Delta_{\mathrm{p}}$ & $2.27 \mathrm{~mm}$ & \\
Horizontal $\frac{1}{2}$ size, $\Delta$ & $7.0 \mathrm{~mm}$ & $\Delta=\Delta_{\beta}+\Delta_{\mathrm{P}}$ \\
Circumference factor, $C_{\mathrm{F}}$ & & $\mathrm{C}_{\mathrm{F}}=1-\frac{3}{4} \mathrm{t}_{\mathrm{O}}$ \\
\hline
\end{tabular}

Note that we have introduced a "circumference factor" diminishing the effect of the induced fields. This is because of the fact that when the beam moves off center, in dispersionless regions, no movement toward the wal1 occurs. Thus, we have introduced a factor,

$$
c_{F}=1-\frac{1}{4} t_{0}
$$

This means that the total induced force decreases as we move off center as part of the beam around the ring does not contribute to the growing oscillation. We take this decreased participation to be linear in the distance from the chamber center, $t_{0}$, and assume that $25 \%$ of the beam is lost from participation at the wall $\left(t_{0}=1\right)$.

The corpling function, $\widetilde{G}\left(t_{0}\right)$, is a function of the beam position within the chamber. For use in computations, we take Eq. (50) multiplied by the circumference factor, $C_{F}$. The threshold $\eta\left(t_{0}\right)$ for a uniform beam is evaluated from (61), while the corresponding frequency shift function, $u_{k}\left(t_{0}\right)$, is obtained from (62). The Case I threshold is plotted in Figure 5. The effect of including sin ${ }^{2}$ flanks is seen from the Case II plot of $\eta\left(t_{0}\right)$, using the general expression (63) and takiig 20\% tails. The frequency shift functions for cases I and II are plotted in Figure 6 . The e-folding growth rate taken from (59) is valid well above threshold and is plotted in Figure 7.

The single most important feature of these curves is the very strong impact of the wall as the beam position is taken closer and closer. The threshold current drops precipitously as does the e-folding growth time. As the wall effect increases, the oscillation frequency shifts from the high frequency beam edge $\left(u_{R} \sim 1\right)$ to the low frequency edge $\left(u_{R} \sim-1\right)$. This, as can be seen in Figures 5 and 6 , is related to the cancellation of self and image contributions to the reactive part of the impedance [that is, the real yart of $\widetilde{G}$ in Eq. (50)]. This cancellation is 


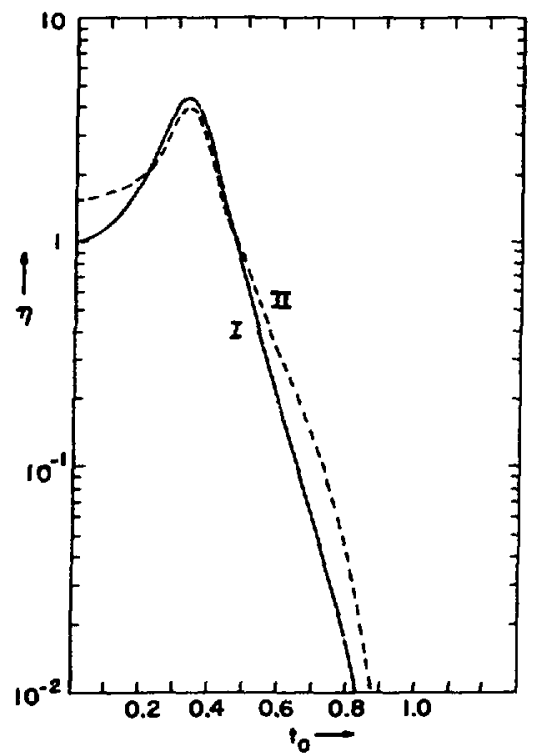

Figure 5. Current threshold function for "small beam" case. $\eta 1 \mathrm{~s}$ normalized to 1 at chamber center with unfform distribution. $t_{0}$ is ratio of distance of $f$ center to chamber radius. I. Uniform density distribution. II. $\sin ^{2}$ flanks: $\alpha_{L}=\alpha_{R}=0.8$.

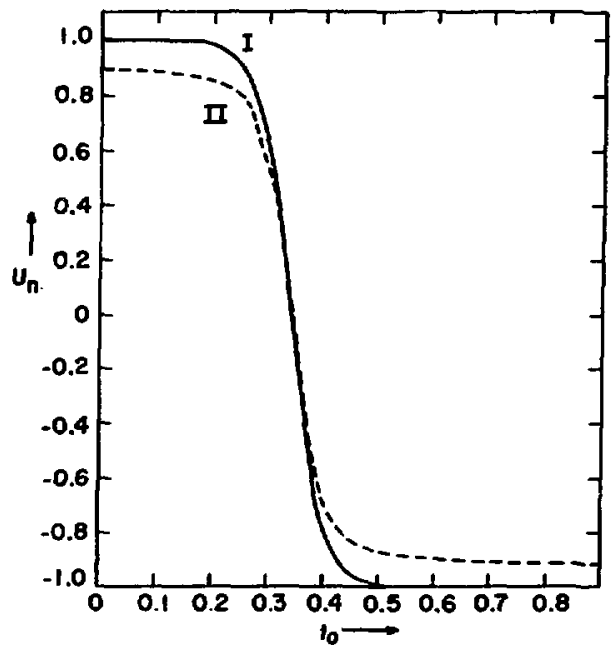

Figure 6. Frequency shift function for "small beam" case. $t_{0}$ is the ratio of distance off center to chamber radius. I. Uniform density distribution. II. $\sin ^{2}$ flanks: $\alpha_{\mathrm{L}}=\alpha_{\mathrm{R}}=0.8$. 


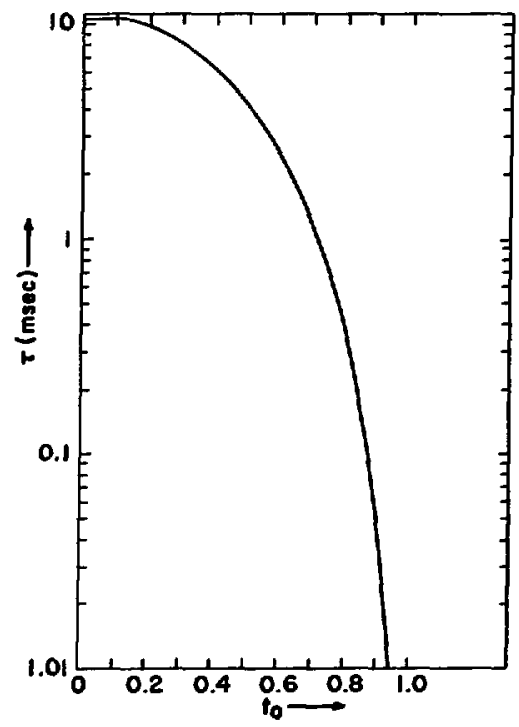

Figure 7. e-folding growth time for a point beam far above threshold. The current $I=1.6 \mathrm{~A}$ (20\% of ISABELLE full current), $T$ is in units of msec. $t_{0}$ is the ratio of the distance off center to the chaniber radius.

reflected in an intermediate rise in current threshold to a peak value as the coherent frequency shifts across the beam.

However, once this cancellation occurs, the wall enhancement becomes dominant and any movement of the beam in the direction of the wall results in a rapid fall of the threshold. From these results, it is clear that the location of "sma11" current stacks in the ISABELLE rings is a critical factor in the design of an operating system for ISABELLE. It is interesting that "small" stacks tend to be less stable than higher current stacks. This will be considered in a later section where results for wide beams are given.

C. Coherent Instability During the Stacking Process

In the process of accumulating current in a proton storage ring br the phase displacement or ISR technique, the beam widens significantly. The stacking scenario is sketched in Figure 8. 


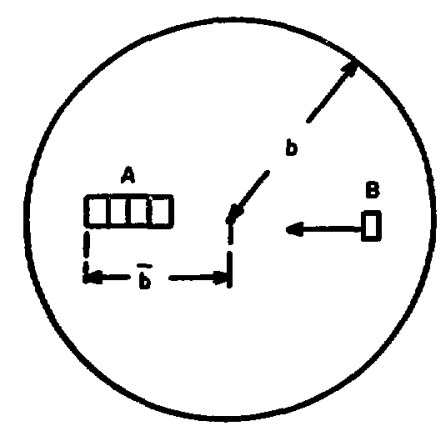

Figure 8. Model stacking process. A: The accumulating stack. B: A small current unit to be added to the existing stack. $b$ is the chamber radius. $\bar{b}$ is the maximum position in the vacuum chamber allowed for beam occupation.

When the stack is complete we have a beam that is shifted to one side of the chamber and occupying a significant fraction of the horizontal aperture. In other words, part of the beam lies zery close to the wall while another part is near the chamber center. Because of the sensitivity of the instability to chamber location, the averaging process which leads to the "point beam" approximation becomes invalid. Thus, we must solve the integral equation (67) and abandon the approximation (50) which led to the dispersion relation (57). To accomplish this, we use the extended Simpson's rule method developed in Section 2.I. The $G$ function to be used can be found from (30) and (49):

$$
\begin{aligned}
G\left(u, u^{\prime}\right)=\left(\frac{I}{e d}\right) \frac{r}{\gamma w_{0} k_{0}} & {\left[\frac { 1 } { \beta ^ { 2 } \gamma ^ { 2 } } \left\{-\frac{1}{\left(x-x^{\prime}\right)^{2}+h^{2}}\right.\right.} \\
& \left.\left.+\frac{b^{2}}{\left(b^{2}-x x^{\prime}\right)^{2}}\right\}+\frac{(1+i) \delta b^{4}\left(b^{2}+x x^{\prime}\right)}{b^{3}\left(b^{2}-x x^{\prime}\right)^{3}}\right],
\end{aligned}
$$

with $x\left(\right.$ or $\left.x^{\prime}\right)$ related to $u\left(\right.$ or $\left.u^{\prime}\right)$ by

$$
x=x_{0}+u \Delta,
$$


while the complex skin depth $\delta$ is given in (21).

Recall that one of the underlying assumptions we have been applying is the linear correspondence between frequency (i.e., tune) and beam horizontal location within the chamber. Thus, we do not differentiate between position due to momentum differences and position due to different betatron amplitudes. In the small beam limit we simply average over the total horizontal extent of the beam and the question ci whether we are dealing with betatron size or momentum size does not arise. Here we must face the question. However, it is clear that any attempt to include the different tune variations due to betatron amplitude and momentum dispersion would be very complicated and for our purposes here it is quite unnecessary. We, therefore, neglect the differentiation and simply retain the linear variation of tune with beam position across the entire beam. By judiciously choosing beam center and beam extent, we can still hope to achieve a valid simulation of the conditions during stacking.

Because of the different underlying assumptions, the "point beam" approximation and the full integral equation solution do not give the same results. We show this in Figure 9 where we compare the threshold

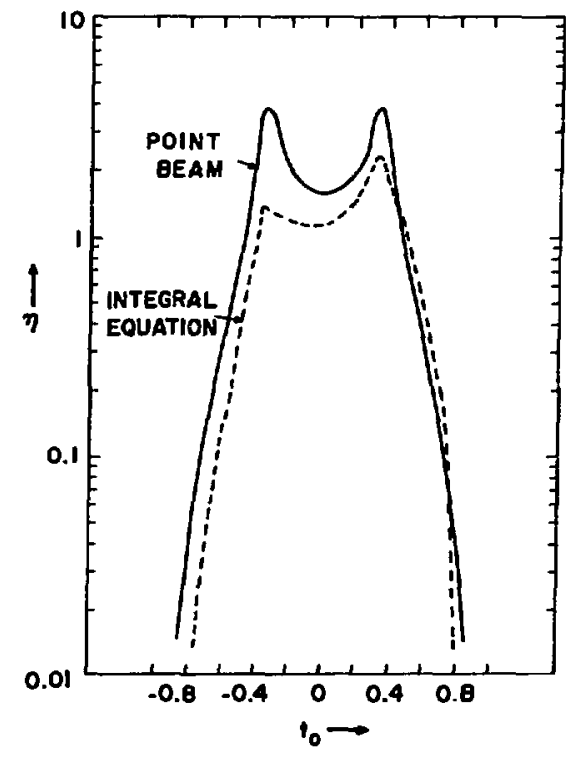

Figure 9. Integral equation solution for threshold function in the case of a "small beam". Comparison with "point beam approximation". $\sin ^{2}$ flanks: $\alpha_{L}=$ $\alpha_{R}=0.8$. Beam parameters are given in Table III.

current as a function of bean center, $t_{0}$, for Case II (i..., $\sin ^{2}$ flanks, $\alpha_{L}=\alpha_{R}=0.8$ ) of Section $3 B$ with the integral equation solution for the small beam described in Table III. They are not too different. However, note especially that the full solution introduces $[t,-]$ asymetry in the dependence on $t_{0}$. This is connected with the 
fact that the coherent frequency is related to beam location. If the coherent frequency is on the side of the beam closest to the wa11, the instability is greater (i。e., a lower current threshold). Thus, in Figure 10 we plot the coherent frequency as a function of $t_{0}$ for the

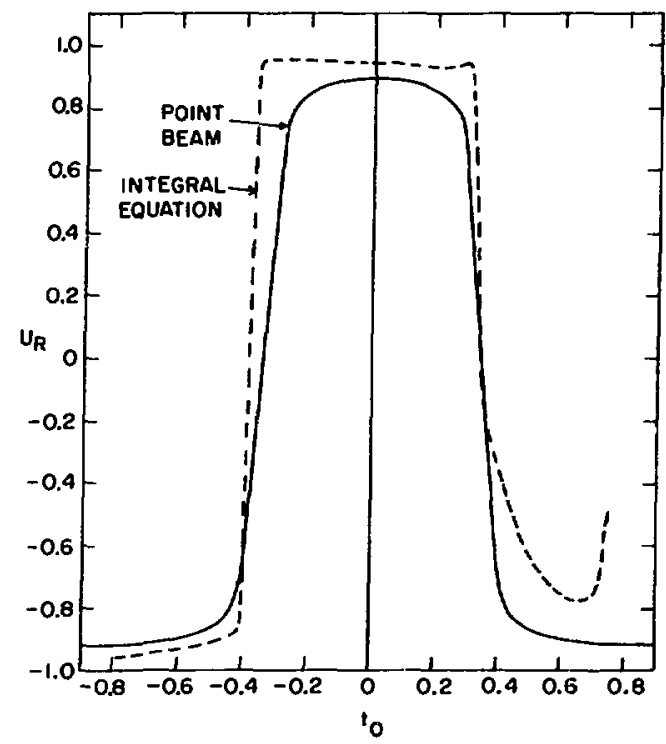

Figure 10. Integral equation solution for frequency shift function in the case of a "small beam". Comparison with "point beam approximation". sin ${ }^{2}$ flanks: $\alpha_{L}=\alpha_{R}=0.8$. Beam parameters are given in Table III.

conditions corr sponding to those in Figure 9. of course, this asymmetry is absent in the "point beam" approximation. The asymmetry effect is even more pronounced in a wide beam, as will be seen below.

In the integral equation solution, there is an extremely rapid shift from the "high frequency" solution to the "low frequency" solution, as can be seen from Figure 10. This is due to the fact that the solutions actually are two distinct modes which appear in the beam with different current thresholds. In Figure 11, we plot $\operatorname{Im}(\eta)$ vs $u_{\mathrm{R}}$ for a few typical values of $t_{o}$. Recall that a solution is physical if $\operatorname{Im}(\eta)=\eta_{I}=0$ and further if $d \eta_{I} / d u_{R}>0$. The high frequency solution is the only mode for small $t_{t_{0}} \mid$. However, for some value of $\left|t_{0}\right|$ a new mode appears at the low frequency end of the beam; Two modes are present with one dominating. For sufficiently large $\left|t_{0}\right|$, the low frequency mode begins to dominate as the enhanced wall term contribution to the impedance becomes larger than the capacitive self-field term. 
ISA $78-12$

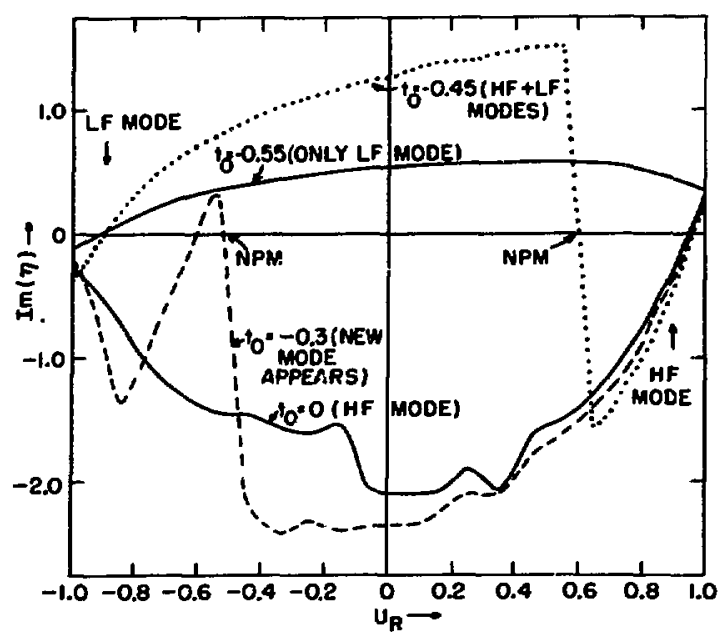

Figure 11. Coherent modes at threshold. HF = High Frequency Mode. $L F=$ Low Frequency Mode. NPM $=$ Nonphysical Mode.

We model the stacking process with 5 steps in the manner sketched in Figure 8. The beam parameters for the 5 steps are listed in Table IV.

Table IV: Beam Parameters for Stacking Procedure

\begin{tabular}{|c|c|c|c|c|c|}
\hline Beam parameter & & & Step Number & & \\
\hline & 1 & 2 & 3 & 4 & 5 \\
\hline $\begin{array}{l}\text { Beam current, I (A) } \\
\text { Tune bpread, 2d }\end{array}$ & $3.68 \times 10^{-3.6}$ & $7.36 \times 10^{3.2}$ & $\begin{array}{r}4.8 \\
1.10 \times 10^{-2}\end{array}$ & $\begin{array}{r}6.4 \\
1.47 \times 10^{-2}\end{array}$ & $\begin{array}{r}8.0 \\
1.84 \times 10^{-2}\end{array}$ \\
\hline $\begin{array}{l}\text { Nominal tune density } \\
\left(i_{0}=I / 2 d\right) \\
\text { Far beam edge (cm) }\end{array}$ & $\begin{array}{r}433.5 \\
3.6\end{array}$ & $\begin{array}{r}433.5 \\
3.6\end{array}$ & $\begin{array}{r}433.5 \\
3.6\end{array}$ & $\begin{array}{r}433.5 \\
3.6\end{array}$ & $\begin{array}{r}433.5 \\
3.6\end{array}$ \\
\hline Horizontal $\frac{1}{2}-\operatorname{size}(\mathrm{cm})$ & 0.70 & 0.93 & 1.15 & 1.38 & 1.61 \\
\hline Beam center, $x_{0}(\mathrm{~cm})$ & 2.90 & 2.67 & 2.45 & 2.22 & 1.99 \\
\hline Beam centex, $t_{0}=x_{0} / b$ & 0.66 & 0.61 & 0.56 & 0.50 & 0.45 \\
\hline Near beam edge & 2.20 & 1.74 & 1.30 & 0.84 & 0.38 \\
\hline
\end{tabular}


In Table $V$, we list the current thresholds and eoherent frequencies Table V: Wide Beam Thresholds During Stacking*

\begin{tabular}{|c|c|c|c|c|}
\hline \multirow{2}{*}{$\begin{array}{l}\text { Step } \\
\text { No. }\end{array}$} & \multicolumn{2}{|c|}{$x_{0}>0$} & \multicolumn{2}{|c|}{$x_{0}<0$} \\
\hline & $\begin{array}{c}\text { Threshold } \\
7\end{array}$ & $\begin{array}{c}\text { Coherent frequency } \\
\mathbf{u}_{R}\end{array}$ & $\begin{array}{c}\text { Threshold } \\
\pi\end{array}$ & $\begin{array}{c}\text { Coherent frequency } \\
u_{R}\end{array}$ \\
\hline 1 & $0 . .80$ & -0.777 & 0.068 & -0.939 \\
\hline 2 & 0.384 & -0.664 & 0.077 & -0.944 \\
\hline 3 & 0.478 & -0.538 & 0.088 & -0.948 \\
\hline 4 & 0.607 & -0.434 & 0.110 & -0.951 \\
\hline 5 & 0.664 & -0.286 & 0.120 & -0.953 \\
\hline
\end{tabular}

for the 5 steps in the case of stacking with $x_{0}<0$.as well as for the case with $x_{0}>0$. The strong enhancement effect for off-center beams is evident from the reduction in tive current threshold. This is especially true for the initial stage of the stacking cycle. We see also the striking asymetry. Stacking in the negative $x_{0}$ region implies that the coherent frequency is near the low frequency edge of tne beam which corresponds to that part of the beam nearest the wal1. Thus, there is a very strong enhancement effect. On the other hand, stacking in the positive $x_{0}$ region means that the part of the beam driven most strongly will be away from the edge close to the chamber wa11. The enhancenent is, therefore, substantially reduced.

\section{CONCLUSIONS}

We have extended the analysis of Laslett, Neil, and Sessler ${ }^{1}$ of transverse coherent beam oscillations to include the case of noncentered and "wide" beams. We have found that off-axis beams in a circular chamber experience a strong enhancement of the resistive wall instability. In particular, we have analyzed the behavior of ribbonlike beams in a circular chamber. This geometry is pertinent to the ISABELLE design ${ }^{2}$ during the current accumulation phase. Introducing a model of the ISABELLE beam, we have attempted to simulate the stacking process.

From our results we can conclude that the stacked beam will be most vulnerable at the beginning of the process. Secondly, we find that stacking in the low frequency region $\left(u_{R} \sim-1\right)$ of the vacuum chamber $\left(x_{0}<0\right)$ reduces the threshold current density by more than an order of magnitude. This suggests that the stacked beam he kept away

1. L.J. Laslett, V.K. Neil, and A.M. Sessler, Rev. Sci. Instrum. 36, 436 (1965).

2. ISABELIE-A Proton-Proton Colliding Beam Facility, BNL 50718, 1978. 
from the low frequency (i.e., low momentum) side of the chamber. In practical terms this means that injection should take place at the low momentum edge and that the injected beam be accelerated into the stack situated in the high momentum region of the chamber. However, even in this case, the initial part of the stack will have a reduction in current density from the nominal value of about a factor of 4 . Also, we remark that the stability of the injected bunches has rot been considered. Since, from the above argument, it would appear preferable to inject from the inside of each ISABELLE ring, i.e., in the low frequency part of the chamber, we feel that the vulnerability of the injected bunches to low frequency transverse oscillations should be studied. It is clear that more "damping" is needed for the ISABELLE beam during stacking. Increasing the tune spread with a chromaticity increase is not very attractive since then a very large tune aperture would be needed to accommodate both the injected beam and the stack. Direct feedback also has complications in that the e-folding growth times tend to be quite short - perhaps as low as a msec - and both stack and injected bunches must be dealt with simultaneously.

Finally, we should point out that only tie resistive wall has been considered here. At low frequencies, we expect that wall discontinuities will contribute an inductive component to the impedance and we further expect an off-center enhancement for the added inductive coupling term. Thus, we suggest that the impact of an inductive wall be studied as an addition to the enhanced resistive wall.

\section{ACKNOWLEDGMENTS}

We would like to express our thanks to J.C. Herrera and R.F. Peierls for fruitful discussions on various aspects of the work presented here. We would also like to thank W.T. Weng for editing parts of the manuscript.

\section{Distribution: External}

